The Astrophysical Journal, 673: L151-L154, 2008 February 1

(C) 2008. The American Astronomical Society. All rights reserved. Printed in U.S.A.

\title{
THE SPITZER GOULD BELT SURVEY OF LARGE NEARBY INTERSTELLAR CLOUDS: DISCOVERY OF A DENSE EMBEDDED CLUSTER IN THE SERPENS-AQUILA RIFT
}

\author{
R. A. Gutermuth, ${ }^{1}$ T. L. Bourke, ${ }^{1}$ L. E. Allen, ${ }^{1}$ P. C. Myers, ${ }^{1}$ S. T. Megeath, ${ }^{2}$ B. C. Matthews, ${ }^{3}$ J. K. Jørgensen, ${ }^{4}$ \\ J. Di Francesco, ${ }^{3}$ D. Ward-Thompson, ${ }^{5}$ T. L. Huard, ${ }^{1}$ T. Y. Brooke, ${ }^{6}$ M. M. Dunham, ${ }^{7}$ \\ L. A. Cieza, ${ }^{7}$ P. M. Harvey, ${ }^{7}$ And N. L. Chapman ${ }^{8}$ \\ Received 2007 November 21; accepted 2007 December 14; published 2008 January 8
}

\begin{abstract}
We report the discovery of a nearby, embedded cluster of young stellar objects, associated filamentary infrared dark cloud, and $4.5 \mu \mathrm{m}$ shock emission knots from outflows detected in Spitzer IRAC mid-infrared imaging of the Serpens-Aquila Rift obtained as part of the Spitzer Gould Belt Legacy Survey. We also present radial velocity measurements of the region from molecular line observations obtained with the Submillimeter Array (SMA) that suggest the cluster is comoving with the Serpens Main embedded cluster $3^{\circ}$ to the north. We therefore assign it the same distance, $260 \mathrm{pc}$. The core of the new cluster, which we call Serpens South, is composed of an unusually large fraction of protostars $(77 \%)$ at high mean surface density $\left(>430 \mathrm{pc}^{-2}\right)$ and short median nearest neighbor spacing (3700 AU). We perform basic cluster structure characterization using nearest neighbor surface density mapping of the YSOs and compare our findings to other known clusters with equivalent analyses available in the literature.
\end{abstract}

Subject headings: ISM: individual (IRAS 18275-0203, IRAS 18274-0205) — stars: formation stars: low-mass, brown dwarfs

\section{INTRODUCTION}

The aim of the Spitzer Gould Belt (GB; L. Allen et al., in preparation) and c2d (Evans et al. 2003) Legacy Surveys is to build a complete mid-infrared record of star formation in all of the large molecular clouds within $500 \mathrm{pc}$, largely dominated by the Gould Belt itself. This is one of several surveys of the Gould Belt being performed at many wavelengths from the near-IR (Two Micron All Sky Survey [2MASS]; Skrutskie et al. 2006) through the far-infrared (Herschel; André \& Saraceno 2005 ) to the submillimeter (Submillimeter Common User Bolometer Array 2 [SCUBA-2]; Ward-Thompson et al. 2007). In combination, the resulting database offers a nearly complete and unbiased view of all nearby star formation in large molecular clouds. This penetrating examination of regions that have often received little attention in the literature is likely to reveal new regions of star formation.

The Spitzer Infrared Array Camera (IRAC) imaging presented here reveals a dense cluster of sources with excess infrared emission and associated $4.5 \mu \mathrm{m}$-bright structured nebulosity (typically shocked $\mathrm{H}_{2}$ emission from outflows; see Smith et al. 2006) within a filamentary infrared dark cloud seen in absorption against bright diffuse 5.8 and $8.0 \mu \mathrm{m}$ emission. This emission is most likely PAH-feature emission (Morris et al. 2004) excited by the young, high-mass stars in W40 (Smith

\footnotetext{
${ }^{1}$ Harvard-Smithsonian Center for Astrophysics, 60 Garden Street, Cambridge, MA 02138; rgutermuth@cfa.harvard.edu.

${ }^{2}$ Ritter Observatory, Department of Physics and Astronomy, University of Toledo, Toledo, $\mathrm{OH} 43606$.

${ }^{3}$ Herzberg Institute of Astrophysics, National Research Council of Canada, Victoria, BC V9E 2E7, Canada.

${ }^{4}$ Argelander-Institut für Astronomie, University of Bonn, Auf dem Hügel 71, 53121 Bonn, Germany.

${ }^{5}$ Department of Physics and Astronomy, University of Cardiff, P.O. Box 913, Cardiff CF2 3YB, Wales, UK.

${ }^{6}$ Spitzer Science Center, MC 220-6, California Institute of Technology, Pasadena, CA 91125

${ }^{7}$ Department of Astronomy, University of Texas, Austin, TX 78712.

${ }^{8}$ Department of Astronomy, University of Maryland, College Park, MD 20742.
}

et al. 1985), 20' to the east. We found structured $K_{s}$ nebulosity at the site in 2MASS Atlas images, but few sources.

The region is flanked by two Infrared Astronomy Satellite (IRAS) sources (IRAS 18275-0203 and IRAS 18274-0205), and Midcourse Space Experiment (MSX) coverage of this region reveals only a few additional point sources. No deep X-ray images of this region are available.

\section{OBSERVATIONS}

We obtained 3.6, 4.5, 5.8, and $8.0 \mu \mathrm{m}$ imaging of 5.1 square degrees of the Serpens-Aquila Rift with IRAC (Fazio et al. 2004) on board Spitzer (Werner et al. 2004) as part of the GB Legacy Survey (PID: 30574) on 2006 October 27. In this Letter, we focus on the $14^{\prime} \times 10^{\prime}$ field of view centered on Serpens South (R.A., decl. [J2000.0] $=18^{\mathrm{h}} 30^{\mathrm{m}} 03^{\mathrm{s}},-02^{\circ} 01^{\prime} 58.2^{\prime \prime}$ ). High Dynamic Range mode was used, resulting in 0.4 and $10.4 \mathrm{~s}$ integrations for each of four dithered images at each mosaic position. Mosaics were constructed at $1.2^{\prime \prime}$ pixel $^{-1}$ with Basic Calibrated Data (BCD) products from the Spitzer Science Center's data pipeline version 14.4. All artifact treatment, mosaic construction, point-source detection and aperture photometry, and photometric classification of sources presented here have been performed using custom Interactive Data Language (IDL) routines (Gutermuth et al. 2007). We adopted aperture radii and inner and outer background annulus radii of 2.4", 2.4", and $7.2^{\prime \prime}$, respectively. Point-source flux calibration is discussed in detail in Reach et al. (2005). Field averaged 90\% differential completeness magnitude limits are 14.5, 14.2, 13.2, and 12.0 for the $14^{\prime} \times 10^{\prime}$ submosaics at $3.6,4.5,5.8$, and $8.0 \mu \mathrm{m}$, respectively (Gutermuth et al. 2005). The sensitivity of these data is equivalent to that of the IRAC data presented by Winston et al. (2007) for the Serpens Main cluster, which will be discussed in $\S 4$. IRAC photometry catalogs are band-merged together with a radial matching tolerance of 1 ", and then merged with the 2MASS Point Source Catalog (PSC) at a tolerance of $1^{\prime \prime}$. 


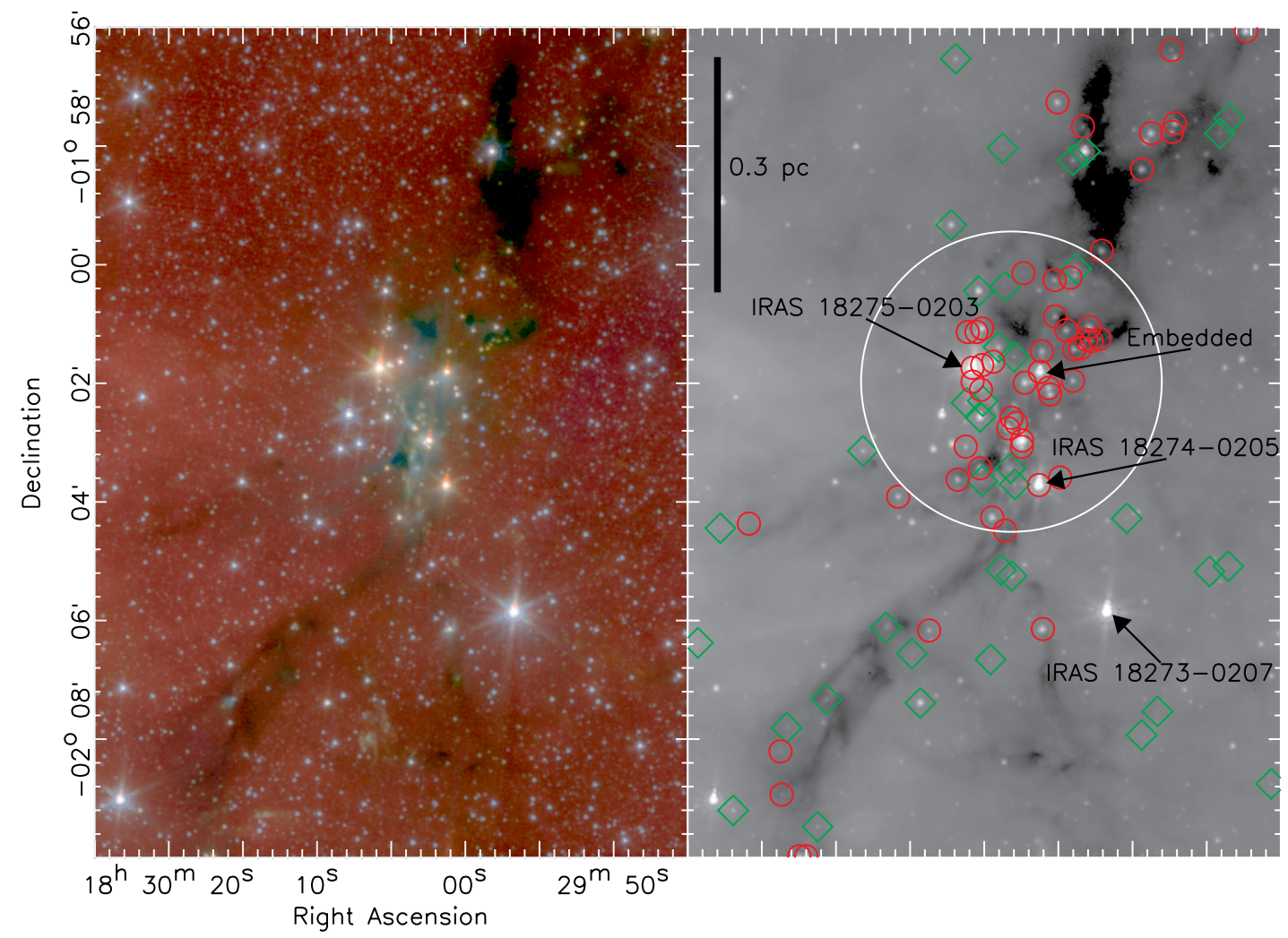

FIG. 1.-At left, a color-composite image of the IRAC mosaics of Serpens South; 3.6, 4.5, and $8.0 \mu \mathrm{m}$ images are mapped to blue, green, and red, respectively. At right, the spatial distribution of YSOs overlaid on the gray-scale $8.0 \mu \mathrm{m}$ image. Red circles are Class I protostars, and green diamonds are Class II stars with disks. The white circle marks the dense core of the cluster.

\subsection{Submillimeter Array}

Observations with the $\mathrm{SMA}^{9}$ (Ho et al. 2004) were performed on 2007 January 30 . The array was tuned to cover the frequency range 219.4-221.4 GHz (LSB) and 229.4-231.4 GHz (USB), which includes the lines of ${ }^{12} \mathrm{CO},{ }^{13} \mathrm{CO}$, and $\mathrm{C}^{18} \mathrm{O} 2-1$, with a resolution of $\sim 0.5 \mathrm{~km} \mathrm{~s}^{-1}$ channel $^{-1}$. Three positions were observed for 30 minutes each: IRAS 18275-0203 (R.A., decl. $\left.[\mathrm{J} 2000.0]=18^{\mathrm{h}} 30^{\mathrm{m}} 05.8^{\mathrm{s}},-02^{\circ} 01^{\prime} 45^{\prime \prime}\right)$ and a nearby embedded source (R.A., decl. [J2000.0] $=18^{\mathrm{h}} 30^{\mathrm{m}} 01.3^{\mathrm{s}},-02^{\circ} 01^{\prime} 48^{\prime \prime}$; labeled "Embedded" in Fig. 1), both in Serpens South, and a position near the ${ }^{12} \mathrm{CO} 1-0$ peak of $\mathrm{W} 40$, as determined from the maps of Grabelsky et al. (1987) at R.A., decl. (J2000.0) = $18^{\mathrm{h}} 31^{\mathrm{m}} 20.7^{\mathrm{s}},-02^{\circ} 01^{\prime} 07^{\prime \prime}$. Ganymede was observed for passband calibration. No flux or complex gain calibration was performed. More detailed SMA results will be discussed in a future paper.

\section{RESULTS}

\subsection{Distance}

Serpens South appears to be associated with a filamentary cloud seen in absorption against PAH emission from W40 (Fig. 1). Thus the distance to W40 (550 \pm 150 pc; Radhakrishnan et al. 1972; Smith et al. 1985) is an upper limit to the distance to Serpens South. The Serpens Main cluster has local standard of rest (LSR) velocities in the range $6-11 \mathrm{~km} \mathrm{~s}^{-1}$, when observed in the isotopologues of CO (White et al. 1995),

\footnotetext{
${ }^{9}$ The Submillimeter Array is a joint project between the Smithsonian Astrophysical Observatory and the Academia Sinica Institute of Astronomy and Astrophysics and is funded by the Smithsonian Institution and the Academia Sinica.
}

with Gaussian FWHM widths of $\sim 2 \mathrm{~km} \mathrm{~s}^{-1}$. Thus, its molecular line emission can be separated from that associated with W40, which occurs in the LSR range of $2-6 \mathrm{~km} \mathrm{~s}^{-1}$ (Zeilik \& Lada 1978; Vallee et al. 1992; Zhu et al. 2006), and is spatially compact. The SMA data clearly show that the velocities of IRAS $18275-0203$ and the nearby embedded source are the same as that of the Serpens Main cluster, and not of the W40 cluster. We therefore assign the distance of $260 \pm 37$ pc (Straižys et al. 1996) to Serpens South. This is consistent with more recent results for the entire Serpens-Aquila Rift (Straižys et al. 2003).

\subsection{Identification, Membership, and Spatial Distribution of YSOs}

We identify likely YSOs from their excess infrared emission over that expected from a typical photosphere and high infrared flux compared to extragalactic contaminants (Gutermuth et al. 2007). These candidates are classified as Class I (protostar) or Class II (pre-main-sequence star with disk) YSOs ${ }^{10}$ by the their value of $\alpha_{\text {IRAC }}$, the linear least-squares fit to their spectral energy distribution ( $\log \lambda F_{\lambda}$ vs. $\log \lambda$ ) through the four IRAC bandpasses (Lada et al. 2006). In this way, we have identified 54 Class I and flat-spectrum "protostars" $\left(\alpha_{\text {IRAC }}>-0.3\right.$; Greene et al. 1994) and 37 Class II $\left(-1.6 \leq \alpha_{\text {IRAC }} \leq-0.3\right)$ YSOs, a total of $91\left(59 \%\right.$ protostars), within the $14^{\prime} \times 10^{\prime}(1.1 \mathrm{pc} \times$ $0.8 \mathrm{pc}$ ) field of view presented in Figure 1. Half of the sources in this field of view are located in a region that stands out as both relatively high in surface density and dominated by

\footnotetext{
${ }^{10}$ Class III (diskless) YSOs cannot be distinguished from field stars in this way (Megeath et al. 2004), but can be effectively identified with X-ray observations (see Feigelson et al. 2007).
} 


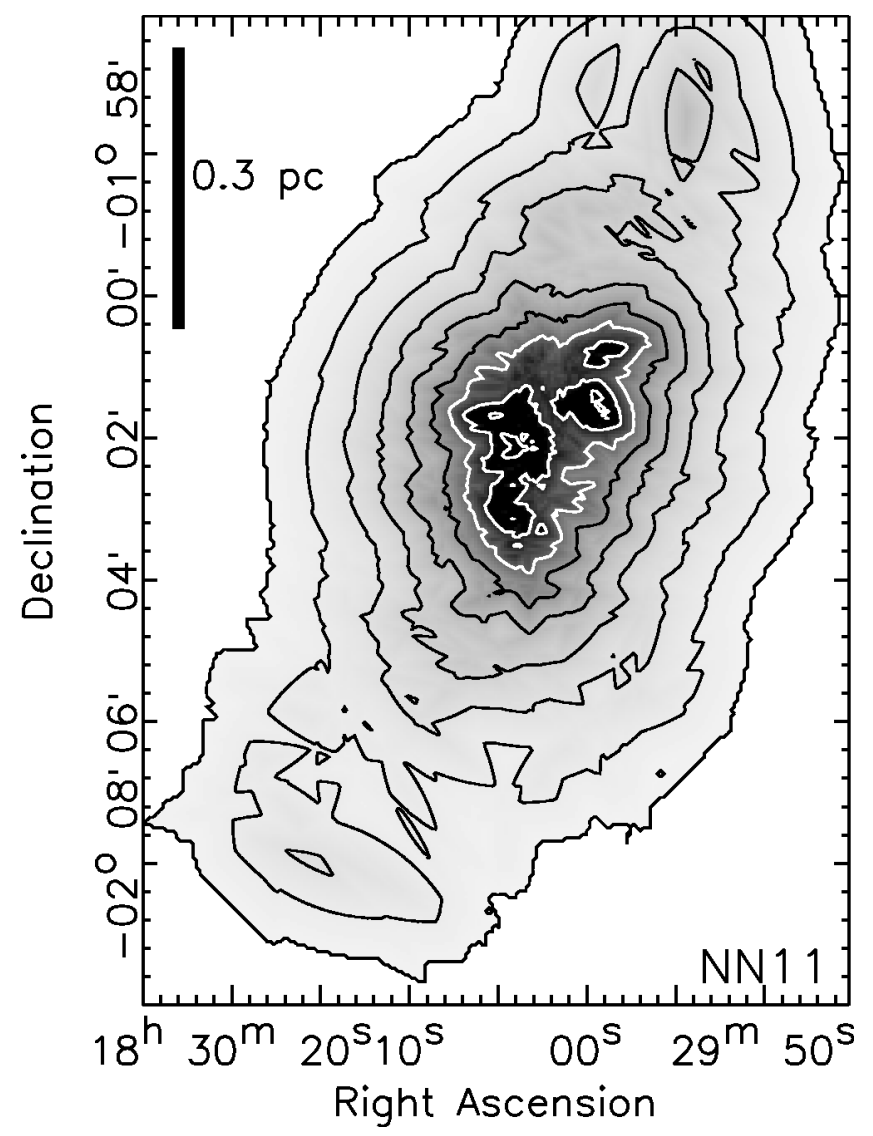

FIG. 2.-A $n=11$ nearest neighbor surface density map for the Serpens South YSOs. Contour levels mark stellar surface densities of $1 \sigma(33 \%)$ below successive contours $\left(1300,890\right.$, and $590 \mathrm{pc}^{-2}$ in white and 400, 260, 180, 78, and $52 \mathrm{pc}^{-2}$ in black), and the linear inverse gray-scale sets $1000 \mathrm{pc}^{-2}$ as black and $0 \mathrm{pc}^{-2}$ as white. The field of view is the same as for Fig. 1.

sources that are protostellar. Of the YSOs identified, 37 Class I and 11 Class II sources are located within a $2.5^{\prime}(0.2 \mathrm{pc})$ radius circle centered on R.A., decl. $(\mathrm{J} 2000.0)=18^{\mathrm{h}} 30^{\mathrm{m}} 03^{\mathrm{s}}$, $-02^{\circ} 01^{\prime} 58.2^{\prime \prime}$, making up the core of the Serpens South Cluster (Fig. 1). In this core, $77 \%$ of the YSOs are protostellar, with a mean density of $430 \mathrm{pc}^{-2}$. This dense grouping appears elongated in an alignment similar to the dust filament seen in absorption ( $150^{\circ}$ east of north). The asymmetric distribution suggests that the mean surface density of $430 \mathrm{pc}^{-2}$ in the core is likely a significant underestimate. To demonstrate this, we note that the median projected distance between nearest neighbor YSOs here is $13.2^{\prime \prime}$, or 3700 AU. Sources uniformly spaced at a density of $430 \mathrm{pc}^{-2}$ are $21.4^{\prime \prime}$ apart, or 5600 AU.

\subsection{YSO Surface Density Mapping}

To characterize the two-dimensional structure of the Serpens South cluster, we have constructed a nearest neighbor surface density map of all the Spitzer-identified YSOs (Fig. 2). The method used to generate these maps is documented in Gutermuth et al. (2005), ${ }^{11}$ although we have used $n=11$ nearest neighbors here to sample the surface densities at higher fidelity (33\% uncertainty; Casertano \& Hut 1985). The elevated density is evident in the center of the map, with a peak density of 1600 $\mathrm{pc}^{-2}$. The apparent boundary contour for the dense cluster core

${ }^{11}$ In summary, from each position in a uniform grid, we measure the distance $r_{n}(i, j)$ that defines a circle that contains the nearest $n$ sources. From this radial distance, a surface density is directly computed as $\sigma_{n}(i, j)=(n-1) / \pi r_{n}^{2}(i, j)$ (Casertano \& Hut 1985).

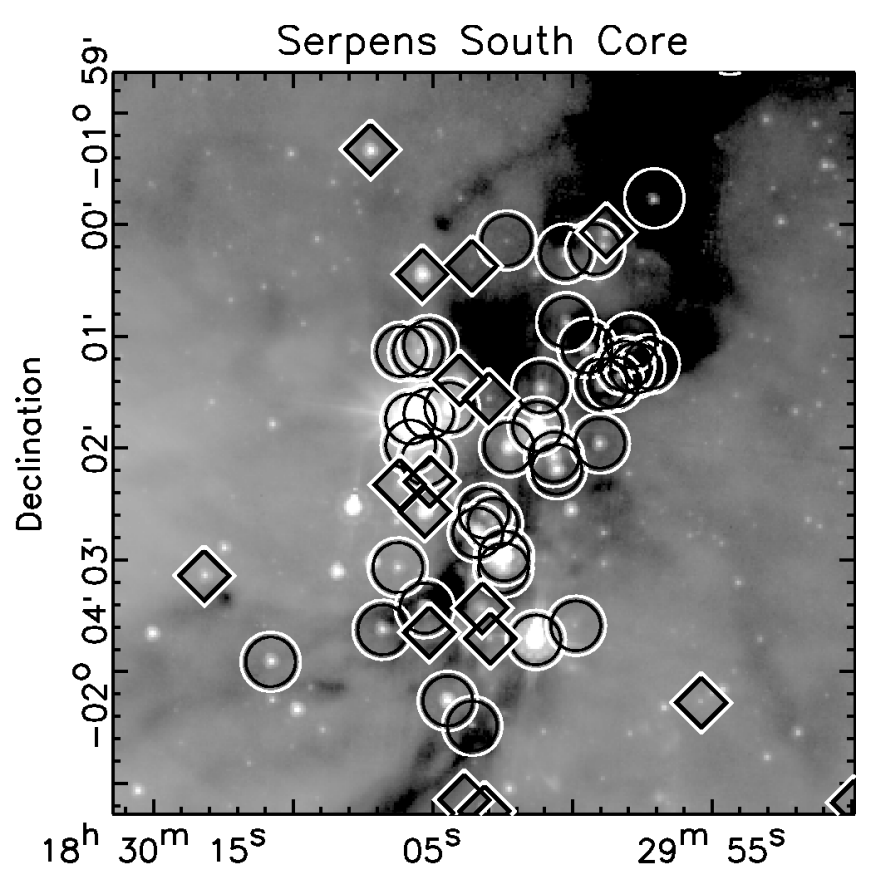

Right Ascension

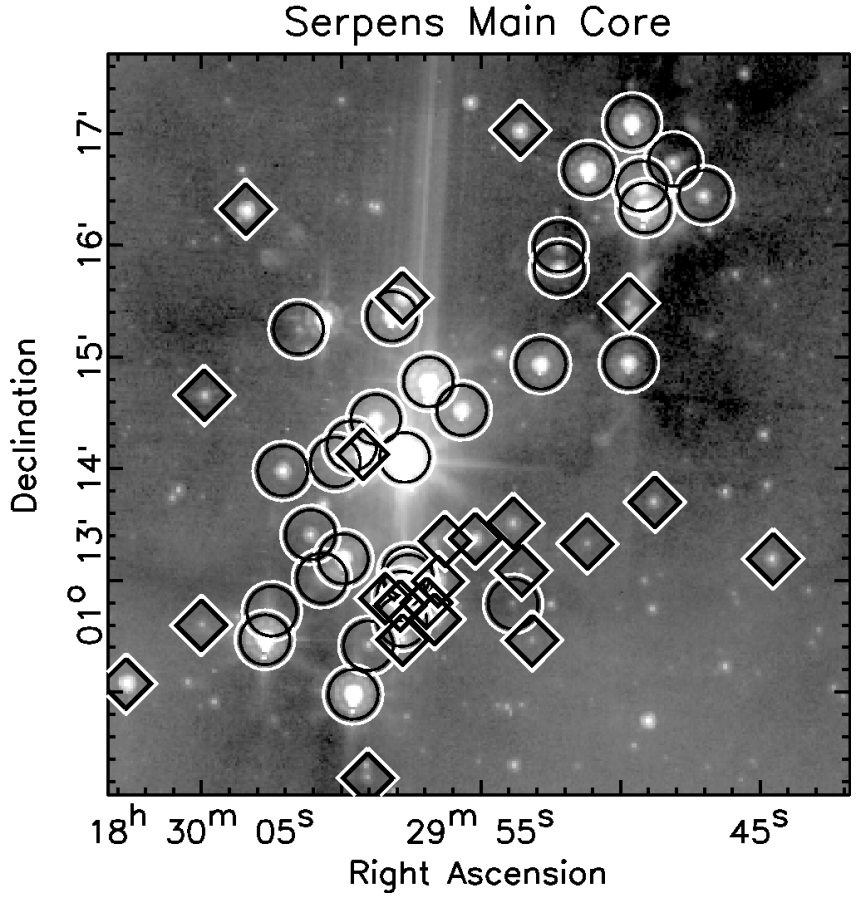

FIG. 3.-Spitzer IRAC $8 \mu \mathrm{m}$ images of the $0.5 \mathrm{pc} \times 0.5 \mathrm{pc}$ core regions of the Serpens South (this work) and Serpens Main (Winston et al. 2007) clusters. Overlaid are the positions of the Class I sources (circles) and the Class II sources (diamonds) for each region. We have included the flat-spectrum sources from Winston et al. (2007) as part of the Class I population for the purposes of this plot.

is $590 \mathrm{pc}^{-2}$, a more reasonable mean surface density than was measured over the poorly matched circular area used in $\S 3.2$ above. Lower density star formation along the dust filaments is also apparent at densities from 50 to $120 \mathrm{pc}^{-2}$. The overall structure is elongated, reflecting the same orientation and extent as the filamentary cloud structures seen in absorption (Fig. 1).

\section{PUTTING SERPENS SOUTH IN CONTEXT}

The Serpens South core's high mean surface density $(>430$ $\mathrm{pc}^{-2}$ ), exceptionally large fraction of Class I sources $(77 \%)$, 
and relatively large membership (48, with 43 more along the $0.5 \mathrm{pc}$ cloud filaments) suggest both a very recent onset of star formation [within the typical lifetime of the protostellar phase, $(2 \pm 1) \times 10^{5} \mathrm{yr}$; see Kenyon \& Hartmann 1995] and high star formation rate $\left(\sim 90 \mathrm{M}_{\odot} \mathrm{Myr}^{-1}\right.$ in the core, assuming the above protostellar phase lifetime and $0.5 M_{\odot}$ per source). Visually, the region bears some resemblance to the Serpens Main cluster (Harvey et al. 2006; Winston et al. 2007) that we have shown lies at a similar distance. In Figure 3, we present 6.5' $\times 6.5^{\prime}(0.5 \mathrm{pc} \times 0.5 \mathrm{pc}) 8 \mu \mathrm{m}$ images of the dense cores of both the Serpens South and Serpens Main clusters, overplotted with the positions of Spitzer-identified Class I and Class II sources (Winston et al. 2007). Over these fields of view, the two regions have nearly identical numbers of YSOs with excess infrared emission and similar protostar fractions (55 at 71\% and 54 at $57 \%$ in Serpens South and Serpens Main, respectively). However, Serpens South is more concentrated and confined to its filament axis than Serpens Main, as evidenced by median nearest neighbor distances between YSOs of 3700 and 4800 AU, respectively. These results suggest that Serpens South is at least as young and as prolific a star-forming region as Serpens Main, although sampling statistics prevent us from making any claims beyond similarity. At larger scales, both Serpens Main and Serpens South are active star-forming sites embedded in larger, less dense, less extinguished distributions of YSOs. Given the high likelihood that both clusters are associated with the Serpens-Aquila Rift and their structural and evolutionary similarity over such a large projected distance from each other $\left(197^{\prime}\right.$ or $\left.15 \mathrm{pc}\right)$, we speculate that the initial conditions of this region play a strong role in defining these qualities.

Serpens South joins a growing number of young clusters that are protostar rich and asymmetrically structured. The more typical asymmetric clusters (e.g., NGC 1333, IRAS 20050+2720, GGD 12-15) have evolved to the point that star formation is active throughout most sites of dense gas in their natal clouds.
This is supported by their large numbers of protostars (active current star formation), their larger numbers of stars with disks (star formation in the recent past), and the distributions of both YSO types that appear to reflect the typically filamentary dense gas distributions (Gutermuth 2005; Allen et al. 2007). Serpens South has a particularly high protostar fraction though $(59 \%$; NGC 1333 is $29 \%$ protostars, excluding transition disks; Gutermuth et al. 2007), and it is part of a dense, dusty cloud filament that is currently forming stars at a rather low surface density compared to the core. If the rest of the filament continues forming stars within the next few $10^{5} \mathrm{yr}$, we speculate that this region could evolve into an asymmetric cluster with a number of sources and protostar fraction similar to those of the aforementioned asymmetric examples. Therefore, the Serpens South cluster could be described as a protocluster, a region with a sufficiently high star formation rate to produce a large number of stars before gas dispersal processes (outflows, radiation, etc.) from the growing cluster membership terminate the process. Given its close proximity, follow-up observations (e.g., to characterize the gas distribution and kinematics) and detailed analysis should offer a new and unique view of the earliest stages of clustered star formation.

We thank T. Dame and E. Winston for providing data from Grabelsky et al. (1987) and Winston et al. (2007) in electronic form. This publication makes use of data products from the Two Micron All Sky Survey, which is a joint project of the University of Massachusetts and the Infrared Processing and Analysis Center/California Institute of Technology, funded by the National Aeronautics and Space Administration and the National Science Foundation. This research has made use of the SIMBAD database, operated at CDS, Strasbourg, France. This work is based in part on observations made with the Spitzer Space Telescope, which is operated by the Jet Propulsion Laboratory, California Institute of Technology under a contract with NASA.

\section{REFERENCES}

Allen, L., et al. 2007, in Protostars and Planets V, ed. B. Reipurth et al. (Tucson: Univ. Arizona Press), 361

André, P., \& Saraceno, P. 2005, in The Dusty and Molecular Universe, ed. A. Wilson (ESA SP-577; Noordwijk: ESA), 179

Casertano, S., \& Hut, P. 1985, ApJ, 298, 80

Evans, N. J., II, et al. 2003, PASP, 115, 965

Fazio, G. G., et al. 2004, ApJS, 154, 10

Feigelson, E., Townsley, L., Güdel, M., \& Stassun, K. 2007, in Protostars and Planets V, ed. B. Reipurth et al. (Tucson: Univ. Arizona Press), 313

Grabelsky, D. A., Cohen, R. S., Bronfman, L., Thaddeus, P., \& May, J. 1987, ApJ, 315, 122

Greene, T. P., Wilking, B. A., André, P., Young, E. T., \& Lada, C. J. 1994, ApJ, 434, 614

Gutermuth, R. A. 2005, Ph.D. thesis, Univ. Rochester

Gutermuth, R. A., Megeath, S. T., Pipher, J. L., Williams, J. P., Allen, L. E., Myers, P. C., \& Raines, S. N. 2005, ApJ, 632, 397

Gutermuth, R. A., et al. 2007, preprint (arXiv:0710.1860)

Harvey, P. M., et al. 2006, ApJ, 644, 307

Ho, P. T. P., Moran, J. M., \& Lo, K. Y. 2004, ApJ, 616, L1

Kenyon, S. J., \& Hartmann, L. 1995, ApJS, 101, 117
Lada, C. J., et al. 2006, AJ, 131, 1574

Megeath, S. T., et al. 2004, ApJS, 154, 367

Morris, P. W., Crowther, P. A., \& Houck, J. R. 2004, ApJS, 154, 413

Radhakrishnan, V., Goss, W. M., Murray, J. D., \& Brooks, J. W. 1972, ApJS, 24,49

Reach, W. T., et al. 2005, PASP, 117, 978

Skrutskie, M. F., et al. 2006, AJ, 131, 1163

Smith, H. A., Hora, J. L., Marengo, M., \& Pipher, J. L. 2006, ApJ, 645, 1264

Smith, J., Bentley, A., Castelaz, M., Gehrz, R. D., Grasdalen, G. L., \& Hackwell, J. A. 1985, ApJ, 291, 571

Straižys, V., Černis, K., \& Bartašiūtè, S. 1996, Baltic Astron., 5, 125 2003, A\&A, 405, 585

Vallee, J. P., Guilloteau, S., \& MacLeod, J. M. 1992, A\&A, 266, 520

Ward-Thompson, D., et al. 2007, PASP, 119, 855

Werner, M. W., et al. 2004, ApJS, 154, 1

White, G. J., Casali, M. M., \& Eiroa, C. 1995, A\&A, 298, 594

Winston, E., et al. 2007, ApJ, 669, 493

Zeilik, M., II, \& Lada, C. J. 1978, ApJ, 222, 896

Zhu, L., Wu, Y.-F., \& Wei, Y. 2006, Chinese J. Astron. Astrophys., 6, 61 